\title{
Mortalidad Por Trauma Cardiaco Penetrante en Bogota, Colombia: Análisis de Factores Asociados
}

\author{
Andrés Isaza-Restrepo, Dínimo José Bolívar-Sáenz, Marcos Tarazona, María Fernanda Jiménez, \\ Fabián Armando Gil Laverde
}

\section{RESUMEN}

Antecedentes: La mayoría de las series de pacientes con heridas de corazón publicadas en la literatura son exclusivamente descriptivas y no suficientemente grandes para obtener conclusiones estadísticas. La prevalencia de agresiones en la población civil de algunos sectores de las ciudades Colombianas es elevada.

Materiales y métodos: Para estudiar la probabilidad de mortalidad en los pacientes que ingresan a salas de cirugía con heridas de corazón se realiza un estudio de casos y controles anidado en la cohorte histórica de 240 pacientes admitidos a salas de cirugía del Hospital Occidente de Kennedy con diagnóstico de herida penetrante de corazón, Entre enero de 1999 y Octubre de 2009.

Resultados: La mortalidad global fue de $15 \%(n=36): 52.9 \%$ (9/17) para los pacientes con herida por arma de fuego y $12.1 \%$ $(27 / 223)$ para los pacientes con herida por arma cortopunzante. Los valores del OR permitieron establecer que las lesiones con arma de fuego, el estado hemodinámico, el grado de la herida, las lesiones concomitantes en tórax y/o abdomen y el requerimiento de una toracotomía resucitativa se asocian con mayor probabilidad de muerte. Los resultados del análisis multivariado muestran que los únicos factores que de forma conjunta se asocian con la mortalidad son la lesión con arma de fuego (OR 22.7; IC 95\% 4.6-112.9), y el estado hemodinámico (fatal: OR 11.4; IC 95\% 1.6-82.0, agónico: 12.8; IC 3.4-48.5, shock profundo: 10.35; IC 2.9-36.4).

Conclusiones: EI trauma cardiaco penetrante en nuestra serie fue causado principalmente por heridas de arma cortopunzante, con una sobrevida similar a otros estudios con este mecanismo predominante. Nuestros resultados están de acuerdo con los parámetros de riesgo de mortalidad previamente propuestos en la literatura y en nuestros pacientes el compromiso del estado hemodinámico al ingreso a cirugia y las lesiones ocasionadas por arma de fuego, demostraron ser predictores significativos de la mortalidad. La presencia de taponamiento cardiaco no tuvo un efecto protector.

Palabras clave: Trauma cardíaco penetrante, Arma cortopunzante, Trauma en Colombia.

\footnotetext{
ABSTRACT

Background: Most studies reported in literature of patients with heart injuries are merely descriptive and not large enough to obtain statistical conclusions. The prevalence of attacks on civilians in some sectors of Colombian cities is high.

Materials and methods: To study the probability of mortality in patients admitted to surgical wards with heart wounds, a case-control study nested in the historical cohort of 240 patients admitted to surgical wards Kennedy Western Hospital with a diagnosis of penetrating wound of the heart, between J anuary 1999 and October 2009 was done.
}

Results: Overall mortality was $15 \%(n=36): 52.9 \%$ (9/17) for patients with gunshot wounds and $12.1 \%$ (27/223) for patients with stab wounds. OR values established that the firearm injuries, hemodynamic status, degree of injury, concomitant injuries in chest and / or abdomen and requiring a resuscitative thoracotomy were associated with increased likelihood of death. The results of the multivariate analysis show that the only factors that are associated with mortality are the gunshot injury (OR 7.22, 95\% Cl 4.6-112.9), and hemodynamic status (fatal: OR 11.4, 95 1.6-82.0\%, agonizing: 12.8, Cl 3.4-48.5, deep shock: 10.35, Cl 2.9-36.4).

Conclusion: Penetrating cardiac trauma in our study was mainly caused by stab weapon wounds with a survival similar to other studies with this predominant mechanism. Our results are consistent with the parameters of mortality risk previously proposed in the literature and in our patients the hemodynamic status on admission to surgery and injuries caused by firearms, proved to be significant predictors of mortality. The presence of cardiac tamponade had no protective effect.

Keywords: Penetrating cardiac trauma, Stab wound, Trauma in Colombia.

How to cite this article: Isaza-Restrepo A, Bolívar-Sáenz DJ , Tarazona M, J iménez MF, Laverde FAG. Mortalidad Por Trauma Cardiaco Penetrante en Bogota, Colombia: Análisis de Factores Asociados. Panam J Trauma Critical Care Emerg Surg 2012;1(2):76-84.

\section{Source of support: Nil}

\section{Conflict of interest: None}

\section{INTRODUCCIÓN}

El trauma originado en hechos civiles y militares representa una grave carga social a nivel mundial, por lo que fue reconocido como problema de salud pública en 1996 por la OMS. ${ }^{1}$ Sus características epidemiológicas están condicionadas por múltiples variables socioeconómicas, particularmente el estado de desarrollo de la sociedad y la presencia de conflictos armados. En Colombia, el trauma constituye la segunda causa de muerte después de las enfermedades cardiovasculares. El Instituto Nacional de Medicina Legal y Ciencias Forenses de Colombia reportó 29.222 necropsias por muerte violenta en el año 2010, que incluye homicidios (38.4 por 100.000 habitantes) y accidentes de tránsito (12.5 por 100.000 habitantes). Se calcula una pérdida potencial de 1'133.863 años de vida para ese año. ${ }^{2}$

Estudios previos evidencian que uno de los segmentos corporales más frecuentemente comprometidos en trauma es el tórax, y que cerca del $6.4 \%$ de los pacientes con

Paper presented in Resident Research Competition, 24th Annual Congress of SPT, Asuncion, Paraguay, November 2011. 
trauma torácico presentan lesiones cardiacas, lo que señala la importancia que tienen estas lesiones en términos de frecuencia. ${ }^{3}$ Se calcula una mortalidad entre $55-82 \%$ antes de recibir atención médica, y hasta un $85 \%$ a pesar de ingresar con signos vitales a un centro hospitalario. ${ }^{4-6}$ Pero además de la mortalidad debemos considerar la frecuencia de posibles lesiones intracardiacas residuales, presentación calculada en $4 \%$ a $56 \%$ de los casos. ${ }^{7,8}$

El trauma civil en nuestro país presenta una mayor frecuencia de agresión con armas cortopunzantes, en contraste con otras series publicadas donde predominan las heridas por arma de fuego. Esto determina unas características particulares de presentación, diagnóstico, tratamiento y resultados. La literatura científica sobre el tema muestra diversas series de pacientes, pero el análisis de factores determinantes del pronóstico de muerte es escaso, dado el tamaño de las mismas y la metodología de los estudios. ${ }^{9,10}$ El objetivo primario de este estudio fue identificar factores predictores de sobrevida en pacientes con trauma penetrante de corazón que ingresan a las salas de cirugía de un centro de referencia de trauma en Bogotá (Colombia), recopila nuestra experiencia con estos pacientes y contribuye al conocimiento sobre el tema.

\section{MATERIALES Y MÉTODOS}

Se diseñó un estudio analítico de casos y controles anidado en una cohorte histórica de pacientes admitidos a salas de cirugía del hospital con diagnóstico de herida penetrante de corazón, entre enero de 1999 y octubre de 2009. El Hospital Occidente de Kennedy es un hospital público de la ciudad de Bogotá, que funciona como centro de referencia para pacientes de trauma. Está localizado en el suroccidente de la ciudad, en una de las localidades que presentan una mayor tasa de agresiones. ${ }^{11}$ Cuenta con un servicio de urgencias general, puerta de entrada de los pacientes relacionados en el presente estudio. La atención inicial está a cargo del cirujano general y de los estudiantes de posgrado (Residentes), presentes durante las 24 horas, todos los días del año. A su ingreso, el paciente se estratifica por su estado hemodinámico según la Clasificación de Ivatury ${ }^{12}$ y la reanimación de los pacientes se desarrolla según los protocolos del Advanced Trauma Life Support (ATLS) del American College of Surgeons. ${ }^{13}$ Los pacientes en estado agónico o aquellos que presentan paro cardiaco presencial, son trasladados inmediatamente a salas de cirugía para realización de una toracotomía resucitativa, aunque algunos cirujanos prefieren la esternotomía. ${ }^{14}$ En nuestro hospital no se realizan toracotomías en el servicio de urgencias pues éste carece actualmente de la infraestructura necesaria para tal fin. Aquellos que presentan inestabilidad hemodinámica
(TA sistólica $\leq 80 \mathrm{~mm} \mathrm{Hg}$ luego de reanimación con 2000cc de cristaloides) o signos de taponamiento cardiaco, ${ }^{15}$ son llevados a cirugía para realización de toracostomía cerrada, esternotomía, o toracotomía (izquierda o derecha) dependiendo de la localización de la herida, de los hallazgos clínicos y de los hallazgos posteriores a la toracostomía si es el caso. Los pacientes hemodinámicamente estables, con heridas localizadas en la zona precordial, ${ }^{13}$ al comienzo de la serie eran llevados protocolariamente a cirugía para realización de una ventana pericárdica subxifoidea con el fin de descartar compromiso del corazón, dado que el Hospital no contaba con servicio permanente de ecocardiografía o ultrasonografía para trauma. Los pacientes con sospecha de herida cardiaca que ingresaron muertos a la institución no se consideraron para este estudio.

El método utilizado para clasificar intraoperatoriamente las heridas cardíacas corresponde al indicado por la American Association for the Surgery of Trauma (AAST) y el comité Organ Injury Scale (OIS), desarrollado en 1994. Dicha escala describe, caracteriza y evalúa el pronóstico sistemático de la severidad del trauma cardiaco en seis grados. Los grados I al VI representan lesiones que aumentan en complejidad, así el tipo VI hace referencia a una lesión irreparable incompatible con la vida. ${ }^{16}$

De los 308 casos registrados en salas de cirugía, se excluyeron 68 casos por las siguientes razones: 22 por tener diagnóstico de herida cardiacas Grado I; 13 porque los registros clínicos fueron insuficientes; y 33 porque los registros no coincidieron con el diagnóstico de herida de corazón. La serie analizada incluyó un total de 240 pacientes. Consignamos los datos obtenidos de las historias clínicas en un instrumento de recolección de información que incluyó las variables demográficas, clínicas, quirúrgicas y de la evolución; la mortalidad fue registrada al egreso hospitalario. Los datos fueron recopilados en MS Excel ${ }^{\circledR} \mathrm{y}$ analizados en el programa estadístico Stata ${ }^{\circledR} 11$.

Inicialmente las variables fueron resumidas empleando estadísticas descriptivas, medidas de tendencia central y variabilidad o frecuencias. Para el análisis de asociación se tomaron como casos los pacientes que murieron antes del egreso hospitalario y como controles los pacientes que egresaron vivos del hospital. El análisis de asociación comprendió dos etapas. En la primera etapa se evaluó la asociación de cada factor (variables demográficas, variables del trauma, variables clínicas y variables de procedimiento médico) con la mortalidad hospitalaria de forma independiente mediante un análisis de asociación bivariado, mediante regresión logística se calculó el OR como medida de asociación y se calculó el correspondiente intervalo de confianza del 95\%. En la segunda etapa se 
evaluó de forma conjunta la asociación de todos los factores con la mortalidad hospitalaria, para lo cual se ajustó un modelo de regresión logística multivarible; se empleó la técnica de selección stepwise con probabilidades de entrada y salida de 0.05 para la obtención del modelo final.

\section{RESULTADOS}

\section{Aspectos Demográficos}

El promedio de edad de los pacientes fue 27.8 años (desviación estándar 9.1 años) y la mayoría fueron hombres (96.3\%). Las heridas por arma corto punzante (HACP) se presentaron en el $92.9 \%$ de los casos, se registraron 17 heridas por proyectil arma de fuego (7.1\%) (HPAF).

\section{Características Clínicas}

Según la clasificación de Ivatury, el estado hemodinámico de los pacientes al ingreso al hospital fue normal en $44.2 \%$ ( $\mathrm{n}=$ $106)$; estaban en shock profundo $34.2 \%(n=82)$; agónicos el $18.3 \%(n=44)$ y $3.3 \%(n=8)$ se presentaron en estado fatal.

El taponamiento cardiaco fue la presentación clínica en $67.1 \%(n=161)$ de los pacientes, y la distribución del taponamiento cardiaco fue $67.3 \%(150 / 223)$ en los pacientes con HACP y $64.7 \%$ (11/17) en los pacientes con HPAF, diferencia que no demostró significancia estadística (valor de $\mathrm{p}=0.82$ ).

El tiempo mediano transcurrido entre el impacto de la herida y el procedimiento quirúrgico fue de 60 minutos (rango intercuartil, 40-150). El promedio del tiempo transcurrido entre el momento de la lesión y el inicio de la cirugía fue menor a 120 minutos en el $73.3 \%$ de los pacientes. De acuerdo al estado hemodinámico de los pacientes el tiempo mediano entre el impacto de la herida $\mathrm{y}$ el procedimiento quirúrgico fueron: 52 minutos (rango intercuartilico, 35-60) en pacientes con estado fatal, 40 minutos (30-60) en pacientes con estado agónico, 60 minutos (40-60) en pacientes con shock profundo, y de 150 minutos (85-315) en pacientes con estado normal; los tiempos medianos fueron estadísticamente significativos con respecto al estado hemodinámico normal $(\mathrm{p}<0.001)$.

Es de resaltar que $22 \%$ de los pacientes clasificados al ingreso como hemodinámicamente estables, se encontraron con taponamiento cardiaco en el momento de la cirugía.

El grado de las heridas según la clasificación OIS-AAST fue: grado II $32.9 \%(\mathrm{n}=79)$, grado III $13.3 \%(\mathrm{n}=32)$, grado IV $29.2 \%(n=70)$, grado V $21.7 \%(n=52)$ y grado VI $2.9 \%(n=7)$.

De los 106 pacientes hemodinámicamente estables al ingreso, $71(67.0 \%)$ presentaron heridas cardiacas grado II, 15 pacientes (14\%) tuvieron lesiones grado IV y 4 (3\%) lesiones grado VI. La totalidad de pacientes que cursaron con shock profundo, (82 pacientes), presentaron principalmente heridas grado III a V. La Tabla 1. Indica el numero y el porcentaje de pacientes que ingresaron de acuerdo al estado hemodinamico de ingreso a salas de cirugía.

En orden de frecuencia, las cavidades cardiacas comprometidas fueron el ventrículo derecho en 50\% ( $\mathrm{n}=$ 124) de los casos, el ventrículo izquierdo en $30 \%(n=74)$, la aurícula derecha en $9 \%(\mathrm{n}=22)$ y la aurícula Izquierda en $4 \%(n=10)$. Hubo lesión simultánea de dos cavidades en 4 pacientes (1.6\%), y dos o más heridas en una misma cavidad en 12 pacientes $(5 \%)$. Nueve pacientes presentaron lesión de arterias coronarias: descendente anterior (6), circunfleja (1), diagonal (1), arteria coronaria no clasificada (1). La mortalidad de estos pacientes fue del $22 \%$.

Las lesiones fueron exclusivamente cardiacas en el $44.2 \%(n=106)$ de los pacientes. Presentaron lesiones asociadas en el tórax 49.2\% $(n=118)$ de los pacientes; en el abdomen en $17.9 \%(n=43)$; toracoabdominales en $0.8 \%(n=2)$. En los casos de heridas por ACP $(n=$ $229)$, el $47 \%(n=108)$ fueron exclusivamente cardiacas, y se asociaron con otras lesiones torácicas en 37\% $(\mathrm{n}=$ 86) de los casos. De los 17 heridos por PAF tan solo tres pacientes tuvieron lesiones exclusivamente cardiacas; uno tuvo lesiones toracoabdominales asociadas; 12 pacientes lesiones asociadas en el tórax, 10 en el abdomen.

\begin{tabular}{|c|c|c|c|c|c|}
\hline \multirow{2}{*}{$\begin{array}{l}\text { Grado } \\
\text { Herida }(n \%)\end{array}$} & \multicolumn{4}{|c|}{ Estado Hemodinamico } & \multirow[t]{2}{*}{ Total } \\
\hline & Fatal & Agónico & Shock pro & Normal & \\
\hline \multirow[t]{2}{*}{ II } & 0 & 0 & 8 & 71 & 79 \\
\hline & 0.00 & 0.00 & 9.76 & 66.98 & 32.92 \\
\hline \multirow[t]{2}{*}{ III } & 1 & 5 & 19 & 7 & 32 \\
\hline & 12.50 & 11.36 & 23.17 & 6.60 & 13.33 \\
\hline \multirow[t]{2}{*}{ IV } & 3 & 21 & 31 & 15 & 70 \\
\hline & 37.50 & 47.73 & 37.80 & 14.15 & 29.17 \\
\hline \multirow[t]{2}{*}{ V } & 3 & 18 & 22 & 9 & 52 \\
\hline & 37.50 & 40.91 & 26.83 & 8.49 & 21.67 \\
\hline \multirow[t]{2}{*}{ VI } & 1 & 0 & 2 & 4 & 7 \\
\hline & 12.50 & 0.00 & 2.44 & 3.77 & 2.92 \\
\hline Total & 8 & 44 & 82 & 106 & 240 \\
\hline
\end{tabular}




\section{Abordaje Quirúrgico}

La ventana pericárdica como método diagnóstico confirmatorio de lesión cardiaca se realizó en el $62.5 \%$ $(n=150)$ de casos. Las incisiones para el abordaje quirúrgico de la lesión fueron: la esternotomía en $63.9 \%(n=152)$ de casos y la toracotomía antero lateral en $36.1 \%(n=86)$, Clamshell (n: 1) y la toracotomía posterolateral en 1 caso. Se realizaron 10 toracotomías resucitativas con clampeo de aorta.

\section{Mortalidad de la Serie}

La mortalidad global en la serie fue $15.0 \%(\mathrm{n}=36)$. La mortalidad en los casos de HPAF fue $52.9 \%(9 / 17)$ y en los casos de HPACP fue de $12.1 \%$ (27/223), diferencia que demostró ser estadísticamente significativa (valor de $\mathrm{p}<$ $0.001)$. Las tasas de mortalidad más elevadas se presentaron cuando el paciente ingresó en estado agónico $(27.3 \%, 12 / 44)$; seguidamente del estado hemodinámico fatal $(25.0 \%, 2 / 8)$ y shock profundo $(23.2 \%, 19 / 82)$. Cuando el paciente ingresó normotenso la tasa de mortalidad fue del 2.8\% (3/106). Las diferencias en mortalidad entre el estado hemodinámico al inicio de la cirugía demostró ser estadísticamente significativo (valor de $\mathrm{p}<0.001$ ).

En relación con el grado de las heridas, la tasa de mortalidad en los casos con heridas grado II fue 2.5\% (2/79 pacientes), $12.5 \%$ (4/32) para las heridas grado III, $20.0 \%$ (14/70) para las heridas grado IV; de 26.9\% (14/52) para las heridas grado $\mathrm{V}$ y $28.6 \%$ (2/7) para las heridas Grado VI. La diferencia de mortalidad entre los grados de heridas cardiacas demostró ser estadísticamente significativa (valor de $\mathrm{p}<0.001$ ). La mortalidad global en casos de taponamiento fue del $21.1 \%(34 / 161)$ y del $2.5 \%$ (2/79) en casos sin taponamiento (valor de $\mathrm{p}<0.001$ ). Se realizaron 10 toracotomías resucitativas con clampeo de aorta, con resultado fatal en 7 casos (70\%). En pacientes con sin toracotomías resucitativas, la tasa de mortalidad fue del $12.7 \%(30 / 236)$ (valor de $\mathrm{p}<0.001)$.

Los pacientes que presentaron lesiones torácicas adicionales $(\mathrm{n}=118)$, tuvieron altas tasas de mortalidad (21.2\%). La mortalidad en pacientes que presentaban únicamente lesiones cardiacas fue de $7.6 \%$ y la de los que presentaron lesiones asociadas de fue de $25.6 \%$.

De los pacientes que fueron llevados a esternotomía $(\mathrm{n}=152)$ fallecieron $8.6 \%(\mathrm{n}=13)$ y de los llevados a toracotomía $(n=86)$, fallecieron el $26.7 \%(n=23)$. El análisis de la mortalidad de acuerdo al tipo incisión utilizada, discriminada por el estado hemodinamico, se encontró que ninguna de las incisiones mostró un efecto protector (Tabla 2). Incluso, un paciente que ingreso a las salas de cirugía en estado fatal, fue llevado a esternotomia media, sobreviviendo a la lesión.

\begin{tabular}{lcc}
\multicolumn{3}{|c|}{ Tabla 2: Mortalidad de acuerdo a la incisión y el estado } \\
$\begin{array}{l}\text { hemodinamico. } \\
\text { hstado }\end{array}$ & $\begin{array}{c}\text { Incision } \\
\text { hemodinamico }\end{array}$ & Toracotomia (\%) \\
\hline esternotomia (\%) & \\
\hline Fatal & 0.0 & 40.0 \\
Agonico & 40.0 & 35.5 \\
Shock profundo & 19.1 & 29.4 \\
Normal & 3.3 & 0.0
\end{tabular}

\section{Evolución Clínica}

La mediana de estancia hospitalaria fue de 6 días (Rango intercuartílico 3-9). Requirieron manejo PO en Unidad de Cuidados Intensivos 184 pacientes, con una mediana de estancia en UCI de 5 días. Los pacientes con HACP requirieron con mayor frecuencia manejo en UCI $(78.0 \%$, 174/223), comparado con los pacientes con HPAF, que requirieron UCI en el 58.8\% (10/17) de los casos, aunque la diferencia no fue significativa estadísticamente $(\mathrm{p}=0.07)$.

El $72.5 \%(n=174)$ de los pacientes presentaron un examen físico postoperatorio normal, entre estos se encuentran 24 pacientes presentaron frote pericardio, "hallazgo considerado normal secundario a la pericardiotomia'. Los hallazgos anormales fueron los soplos en $6.6 \%(n=16)$ de casos. Otros 7 pacientes (3\%) presentaron hallazgos como bradicardia, click sistólico, isquemia y supradesnivel ST en el EKG, extratonos, fibrilación auricular. En 12 pacientes (5\%) se desconocen los hallazgos porque fueron remitidos a otras instituciones de salud.

El ecocardiograma transtorácico postoperatorio se realizo en 94 (39\%) pacientes, los cuales fueron normales en $59 \%$ (56). Del $41 \%$ restante, se encontraron alteraciones de la contractilidad en 19 pacientes y lesiones del tabique Interventricular en 4. Se evidenciaron lesiones valvulares tricúspideas tipo insuficiencia en 13 y lesión de la válvula mitral en 1 paciente. No se registró ningún caso de alteración en las cuerdas tendinosas, músculos papilares, válvula aórtica o pulmonar. Lamentablemente no se cuenta con un mayor seguimiento ecocardiográfico de las heridas cardiacas en nuestra institución para determinar lesiones residuales debido a tres razones: primero, a partir del año 2004 se protocolizo su uso rutinario; segundo, la no disponibilidad permanente de los equipos y tercero, la pobre adherencia de los pacientes al seguimiento ambulatorio y la mayoría correspondían a estratos socioeconómicos bajos.

Sólo cuatro pacientes de la serie requirieron remisión posterior a Cirugía Cardiovascular para evaluación y tratamiento.

\section{Análisis de Asociación Con la Mortalidad Hospitalaria}

Los factores que de forma independiente resultan ser factores de riesgo para la mortalidad son: Lesión con arma de fuego, 
estado hemodinámico, grado de la herida, presentar lesiones de tórax, presentar lesiones abdominales, el taponamiento, la incisión y el clamp aórtico. Los únicos factores que se asocian con la mortalidad como factores protectores son la ventana pericárdica y un tiempo mayor de tres horas desde el impacto de la herida y el procedimiento quirúrgico (Tabla 3). Muestra la asociación de los diversos posibles factores de riesgo para mortalidad en pacientes con heridas cardiacas. La distribución de edad no fue significativamente diferente entre los sobrevivientes y los no sobrevivientes.
El análisis de regresión logística muestra que los factores que de forma conjunta se asocian con la mortalidad son lesión con arma de fuego, el estado hemodinámico, el clamp aórtico y la ventana pericárdica. Este último factor a diferencia de los otros se asocia como factor protector. De acuerdo con este modelo, la probabilidad de morir por una lesión cardiaca penetrante es 12 veces mayor si el paciente ingresa a urgencias en estado fatal, 9 veces si llega en estado agónico y 10 veces si ingresa en estado de shock profundo; comparado con

\begin{tabular}{|c|c|c|c|c|c|c|}
\hline \multirow[t]{2}{*}{ Variable } & \multicolumn{2}{|c|}{ Vivos $(n=204)$} & \multicolumn{2}{|c|}{ Muertos $(n=36)$} & \multirow[t]{2}{*}{ OR } & \multirow[t]{2}{*}{ IC $(95 \%)$} \\
\hline & $\mathrm{n}$ & $\%$ & $\mathrm{n}$ & $\%$ & & \\
\hline Edad, promedio & $27.8(0.4)$ & & $27.6(6.9)$ & & 1.00 & $0.96-1.04$ \\
\hline \multicolumn{7}{|l|}{ Sexo } \\
\hline Femenino & 7 & 3.4 & 2 & 5.6 & 1.66 & $0.33-8.31$ \\
\hline Masculino & 197 & 96.6 & 34 & 94.4 & 1.00 & - \\
\hline \multicolumn{7}{|l|}{ Tipo de arma } \\
\hline Blanca & 196 & 96.1 & 27 & 75.0 & 1.00 & - \\
\hline Fuego & 8 & 3.9 & 9 & 25.0 & 8.17 & $2.90-22.96$ \\
\hline \multicolumn{7}{|l|}{ Estado hemodinámico } \\
\hline Normal & 103 & 50.5 & 3 & 8.3 & 1.00 & - \\
\hline Fatal & 6 & 2.9 & 2 & 5.6 & 11.44 & $1.60-82.02$ \\
\hline Agónico & 32 & 15.7 & 12 & 33.3 & 12.88 & $3.42-48.48$ \\
\hline Shock profundo & 63 & 30.9 & 19 & 52.8 & 10.35 & $2.94-36.41$ \\
\hline \multicolumn{7}{|l|}{ Grado herida } \\
\hline$\|$ & 77 & 37.8 & 2 & 5.6 & 1.00 & - \\
\hline III & 28 & 13.7 & 4 & 11.0 & 5.50 & $0.95-31.7$ \\
\hline IV & 56 & 27.5 & 14 & 38.9 & 9.63 & $2.10-44.05$ \\
\hline V & 38 & 18.6 & 14 & 38.9 & 14.18 & $3.07-65.62$ \\
\hline VI & 5 & 2.4 & 2 & 5.6 & 15.4 & $1.78-133.35$ \\
\hline Lesión tórax & 93 & 45.6 & 25 & 69.4 & 2.71 & $1.27-5.80$ \\
\hline Lesión abdomen & 32 & 15.7 & 11 & 30.6 & 2.37 & $1.06-5.28$ \\
\hline Lesión tórax y abdomen & 1 & 0.5 & 1 & 2.8 & 5.80 & $0.35-94.90$ \\
\hline Taponamiento & 127 & 62.3 & 34 & 94.4 & 10.31 & $2.41-44.11$ \\
\hline \multicolumn{7}{|l|}{ Tiempo desde trauma } \\
\hline Menos de 30 minutes & 12 & 5.8 & 3 & 8.2 & 1.00 & - \\
\hline 30 min. $-<1$ hora & 53 & 26.0 & 14 & 38.9 & 1.05 & $0.26-4.27$ \\
\hline 1 hora $-<2$ horas & 70 & 34.3 & 15 & 41.7 & 0.86 & $0.22-3.42$ \\
\hline 2 hora - $<3$ horas & 14 & 6.9 & 2 & 5.6 & 0.57 & $0.08-4.00$ \\
\hline 3 o más horas & 55 & 27.0 & 2 & 5.6 & 0.14 & $0.02-0.97$ \\
\hline Ventana pericárdica & 140 & 68.6 & 10 & 27.8 & 0.18 & $0.08-0.39$ \\
\hline \multicolumn{7}{|l|}{ Incisión } \\
\hline Esternotomia & 139 & 68.8 & 13 & 36.1 & 1.00 & - \\
\hline Toracotomía AL & 63 & 31.2 & 23 & 63.9 & 3.90 & $1.86-8.20$ \\
\hline Clamp aórtico & 3 & 1.5 & 7 & 19.4 & 16.17 & $3.96-66.07$ \\
\hline Sospecha lesión & 22 & 11.5 & 1 & 20.0 & 1.93 & $0.21-18.07$ \\
\hline
\end{tabular}




\begin{tabular}{|c|c|c|c|c|c|c|}
\hline \multirow[t]{2}{*}{ Variable } & \multicolumn{2}{|c|}{ Vivos $(n=204)$} & \multicolumn{2}{|c|}{ Muertos $(n=36)$} & \multirow[t]{2}{*}{ OR } & \multirow[t]{2}{*}{ IC (95\%) } \\
\hline & $\mathrm{n}$ & $\%$ & $\mathrm{n}$ & $\%$ & & \\
\hline \multicolumn{7}{|l|}{ Tipo de arma } \\
\hline Blanca & 196 & 96.1 & 27 & 75.0 & 1.00 & - \\
\hline Fuego & 8 & 3.9 & 9 & 25.0 & 22.7 & $4.6-112.9$ \\
\hline \multicolumn{7}{|l|}{ Estado hemodinámico } \\
\hline Normal & 103 & 50.5 & 3 & 8.3 & 1.00 & - \\
\hline Fatal & 6 & 2.9 & 2 & 5.6 & 12.03 & $1.32-109.42$ \\
\hline Agónico & 32 & 15.7 & 12 & 33.3 & 9.03 & $1.77-46.10$ \\
\hline Shock profundo & 63 & 30.9 & 19 & 52.8 & 9.83 & $2.20-43.89$ \\
\hline Ventana pericardica & 140 & 68.6 & 10 & 27.8 & 0.30 & $0.11-0.39$ \\
\hline Clamp aórtico & 3 & 1.5 & 7 & 19.4 & 8.10 & $1.40-47.03$ \\
\hline
\end{tabular}

un paciente hemodinámicamente normal (La Tabla 4). Muestra los OR del análisis multivariado.

\section{DISCUSIÓN}

A diferencia de lo que ocurre en otros países del mundo, las lesiones cardiacas penetrantes en Colombia son relativamente frecuentes. Entre 1980 y 1984 ingresaba al Hospital San Juan de Dios de Bogotá un paciente con herida cardiaca penetrante cada 48 horas, ${ }^{17}$ en el Hospital San Vicente de Paúl de Medellín, ingresaron entre 1991 a 1996, 776 pacientes con heridas cardiacas penetrantes. ${ }^{18}$ Este estudio representa nuestra experiencia en el manejo del trauma cardiaco penetrante de 240 pacientes durante diez años. Sin embargo, es importante comentar que el numero de pacientes admitidos con trauma cardiaco penetrante ha disminuido en Colombia y particularmente en el Hospital de Kennedy como también en el H San Vicente de Paúl en la ultima década. Atribuimos parcialmente este descenso del trauma penetrante, a partir de la adopción en Bogota de la ley de restricción de expendio de alcohol en espacios públicos que empezó a regir desde 1995.

El Hospital Occidente de Kennedy es un hospital de III nivel de la red hospitalaria de la ciudad de Bogotá, que en la práctica funciona como un centro de referencia para pacientes de trauma en la zona sur-occidental de la ciudad. Cubre una población aproximada de dos millones de habitantes (Bogota metropolitana tiene aproximadamente 9 millones de habitantes). Estudios previos realizados en el hospital muestran que se atienden aproximadamente 30 heridas penetrantes de corazón al año. Las limitaciones tecnológicas propias de hospitales de estas características puede ser determinantes de las conductas para su diagnostico, tratamiento quirúrgico, seguimiento y de los resultados de las mismas. No cuenta permanentemente con un cirujano cardiovascular. Por lo tanto, todos los pacientes de este estudio fueron atendidos por los residentes o los cirujanos generales. Aunque la atención prehospitalaria en Bogota ha mejorado significativamente durante los últimos años, todavía es frecuente encontrar que los pacientes ingresan por sus propios medios o traídos por la policía sin recibir una correcta atención prehospitalaria.

Un alto porcentaje de pacientes (22\%) ingreso en aparente estado hemodinámico normal y a pesar de no mostrar síntomas de taponamiento cardiaco, intraoperatoriamente presentaron heridas III-V. Esto alerta la importancia de realizar activamente y en forma expedita estudios para descartar estas lesiones. En nuestro hospital la ventana pericárdica es el método confirmatorio de trauma cardiaco penetrante, la cual se realizo en el $62.5 \%$ de los casos. Obviamente la presencia de lesiones más complejas según la clasificación del OIS-AAST se correlaciona con un mayor índice de mortalidad, pues cada grado representa un compromiso anatomo fisiológico mayor. Nuestros resultados están de acuerdo con los parámetros previamente propuestos y en nuestros pacientes el compromiso del estado hemodinámico al ingreso, las lesiones ocasionadas por arma de fuego y las lesiones cardiacas con un grado de III a VI, demostraron ser predictores significativos de la mortalidad. En relación con el grado de las heridas, la tasa de mortalidad en los casos con heridas grado II fue $2.5 \%$ (2/79 pacientes), $12.5 \%$ (4/32) para las heridas grado III, 20.0\% (14/70) para las heridas grado IV; de 26.9\% (14/52) para las heridas grado V y $28.6 \%$ (2/7) para las heridas grado VI. La diferencia de mortalidad entre los grados de heridas cardiacas demostró ser estadísticamente significativa (valor de $\mathrm{p}<0.001$ ).

Está demostrado que la condición fisiológica en el trauma cardiaco penetrante está asociado con el mecanismo de lesión. ${ }^{19}$ El principal mecanismo de lesión fueron las armas cortopunzantes $(92.9 \%)$, y a pesar que la diferencia resulto ser estadísticamente significativa $52.9 \%$ armas de fuego vs. 
$12.1 \%$ cortopunzantes $(\mathrm{p}<0.001)$; este aparente exceso no pueden atribuirse a este mecanismo independientemente que ocasionen un mayor daño tisular, se explica porque más de la mitad de los pacientes con heridas por arma de fuego fallecieron $(9 / 17)$ y el poco número de casos. Los heridos con armas de fuego, presentaron 8 veces más chance de morir que aquellos con heridas por arma corto punzante. Esto puede ser secundario a un mayor sangrado o taponamiento con el subsecuente deterioro hemodinámico al ingreso. Similar a lo expuesto por Campbell, et al, Rashid et al, ${ }^{20-22}$ las lesiones torácicas asociadas con hemotórax, son un factor de riesgo de muerte en pacientes con trauma cardiaco penetrante (OR 3 ).

El diagnostico taponamiento cardíaco requiere un alto índice de sospecha basado en la localización de la herida y la presencia de los signos anteriormente descritos. Sin embargo existen lesiones cardiacas penetrantes en ausencia de estos signos, ya sea por hemorragia intratoracica o heridas miocárdicas pequeñas que dejan de sangrar en el momento que hay respuesta a la reanimación. Fisiopatológicamente, el taponamiento presenta tres fases, las dos primeras podrían proteger al paciente contra la exanguinación, pero en la última es causante de arresto cardíaco por compromiso del llenado diastólico y la perfusión miocárdica. En ese orden, el efecto "protector" que se atribuye al taponamiento cardíaco es limitado y tiempo dependiente, los cuales se presentan durante la primera fase y tal vez parte de la segunda. El problema es que hasta el momento no se sabe la duración de cada una de dichas fases y a partir de qué momento se convierte en un factor en contra. En nuestro estudio el $67 \%$ de los casos presento taponamiento, asociándose a 10 veces más riesgo de muerte, sin embargo y como en otras series, ${ }^{9}$ este aparente efecto protector no fue encontrado y a diferencia de otras en donde los pacientes sobreviven más, ${ }^{23}$ la presencia de dicha entidad clínica no fue estadísticamente significativa. En el análisis de regresión, la presencia de taponamiento por sí solo no predijo sobrevida y para evaluar su posible efecto se debe comparar en sujetos con el mismo estado hemodinámico antes del procedimiento quirúrgico. El $22 \%$ de los pacientes que ingresaron normotensos a la sala de operaciones, presentaron intraoperatoriamente diagnostico de taponamiento cardiaco y aunque la mayoría tuvieron heridas grado II, 19 pacientes presentaron lesiones grado IV y grado VI. Este hallazgo es de vital importancia por que explicaría la alta presencia de esta característica clínica en nuestra serie de casos.

En nuestra cohorte la presencia de lesiones asociadas en el tórax fue de 49.2\% $(\mathrm{n}=118)$; en el abdomen de $17.9 \%$ $(n=43)$; encontrando que la presencia de lesiones asociadas en tanto en tórax como en abdomen aumento el chance de morir en casi 6 veces. Las lesiones fueron exclusivamente cardiacas en el $44.2 \%(n=106)$ de los pacientes posiblemente porque el principal mecanismo de trauma de las heridas cardiacas fue la herida por arma cortopunzante. La mortalidad en nuestra serie de heridas toracoabdominales fue de $25.6 \%$, requiriendo adicionalmente laparotomía, la cual es similar a lo reportado por Degiannis y colaboradores, ${ }^{25}$ pero es mejor que la reportada por Asensio y colaboradores $(59 \%)$, por mecanismos de lesiones similares, ${ }^{9}$ sin embargo debe haber claridad que en dichas poblaciones predominan las lesiones por armas de fuego.

Aunque la toracotomia de resucitación realizada en urgencias es preconizada por el Advanced Trauma Life Support para pacientes con trauma penetrante de torso que llegan a urgencias sin pulso pero con actividad eléctrica , anotamos que en nuestro hospital no es posible realizar toracotomías en el servicio de urgencias ya que éste carece actualmente de la infraestructura necesaria. Sin embargo, el tiempo aproximado entre la sala de urgencias y la sala de operaciones es menor a $2 \mathrm{~min}$. Por lo tanto, todas la victimas que se encontraron en estado fatal o agónico fueron rápidamente transferidas a salas de cirugía, con una mortalidad del $70 \%$. Esta medida no tuvo un efecto perjudicial ya que este porcentaje es similar al $74 \%$ descrito por Asensio en 1998. ${ }^{9}$ Es por esto que consideramos que mientras no contemos con una sala de reanimación de trauma en el interior del servicio de urgencias, debemos continuar con dicha estrategia de traslado rápido.

Al igual que el estudio de Mitchell, 1993, Degiannis, 2006 y colaboradores; ${ }^{24,25}$ la esternotomía es el abordaje quirúrgico preferido en nuestra institución. El acceso al mediastino anterior y medio, es rápido y seguro, permite una adecuada exposición del corazón, carina, vasos imnominados y carótida derecha y brinda el espacio para realizar maniobras en trauma tales como la de Sauerbruch (clampeo de ambas cavas) y luxación del corazón para suturas cardíacas complejas. La esternotomía media se realizo en el $63.9 \%(n=152)$ y la toracotomía antero lateral en el $36.1 \%(n=86)$, y a pesar de que los pacientes a quienes se les realizo esta última, presentaron un OR: 4, la relación entre mortalidad, estado hemodinámico al ingreso y tipo de incisión utilizada, no mostró diferencias significativas. (Tabla 2). Incluso en un paciente que se encontraba en estado hemodinamico clasificado como fatal, se realizo la esternotomia media como vía de acceso a la lesión cardiaca, logrando su sobrevida. Aunque otros centros en Colombia y en el mundo utilizan la toracotomía anterolateral izquierda ${ }^{26}$ para todos los casos, nuestra serie reserva este abordaje en pacientes inestables o con arresto cardiaco presenciado, además que facilita el clampeo de la aorta descendente.

La mortalidad global fue 15\% $(n=36)$. Esta tasa es baja a diferencia de otras reportadas la literatura ${ }^{6,9,12}$ y similar a 
la descrita en otras series nacionales $(10.4 \%$ por VillegasMorales y $13 \%$ por Duque). Esto probablemente se debe a que el porcentaje lesiones por arma de fuego es menor que el descrito en otras series y al hecho de haber excluido los pacientes que fallecieron durante la atención inicial en urgencias.

Diversos índices de fisiológicos y el mecanismo de trauma han sido propuestos como predictores de mortalidad. Entre los factores aceptados que afectan la sobrevida, luego de una lesión penetrante al corazón, se han incluido: el tipo de arma utilizada, el tamaño de la lesión miocárdica, la cámara cardiaca lesionada, el compromiso de arterias coronarias, el estado hemodinámico inicial, las lesiones asociadas y el tiempo transcurrido en la llegada al hospital. Es claro que la presencia de lesiones más complejas según la clasificación del OIS-AAST se correlaciona con un mayor índice de mortalidad, pues cada grado representa un compromiso anatomo fisiológico mayor. A pesar de una sobrevida cercana al 75\%, no se debe pensar en una supuesta 'benevolencia' de las heridas cardíacas, pues éstos resultados provienen del análisis de pacientes sobrevivientes, y en muchas de las series publicadas no se discrimina adecuadamente el estado hemodinámico al ingreso. Nuestros resultados están de acuerdo con los parámetros previamente propuestos y en nuestros pacientes el compromiso del estado hemodinámico al ingreso, las lesiones ocasionadas por arma de fuego y las lesiones cardiacas con un grado de III a VI, demostraron ser predictores significativos de la mortalidad. El estado hemodinámico al inicio de la cirugía (agónico 27.3\%, fatal $25 \%$, shock profundo $23.2 \%$, normal $2.8 \%$ ) resultaron ser estadísticamente significativos $(\mathrm{p}<0.001)$.

Aunque en el presente estudio no es posible demostrarlo objetivamente, consideramos que la experiencia adquirida por los cirujanos del Hospital a lo largo de los años en la evaluación y el manejo de las heridas cardiacas penetrantes, es un factor determinante del buen desenlace. Un alto porcentaje de pacientes de nuestra serie tenia lesiones cardiacas complejas (67\%). A pesar de estos, nuestra mortalidad global fue de $15 \%$, menor a la descrita en otras series internacionales con mecanismo de trauma predominantemente por arma cortopunzante con mortalidad global de $25 \%$.

El seguimiento clínico intrahospitalario realizado en nuestra serie fue hasta el egreso de la institución. Las difíciles condiciones socioeconómicas de la población, la no disponibilidad continúa de ecocardiografía y el pobre seguimiento ambulatorio clínico hacen que desconozcamos en nuestra serie las lesiones intracardiacas residuales por heridas cardiacas penetrantes. Sin embargo y teniendo en cuenta que los estudios de Villegas ${ }^{18}$ y Demetriades ${ }^{27}$ y colaboradores, reportan defectos valvulares o septales hasta en $19 \%$ a 23 meses, hoy en día en nuestro hospital esta protocolizado el seguimiento estricto de este tipo de pacientes de manera clínica e imagenológica.

El transporte prehospitalario es un predictor de sobrevida $^{28}$ y a pesar que la atención prehospitalaria en Bogotá ha mejorado significativamente durante los últimos años, es muy común que los pacientes ingresen por sus propios medios o traídos por la policía y nuestra serie no analiza dicha duración. Es claro que tiempos largos y la inadecuada reanimación prehospitalaria (reanimación agresiva con cristaloides) se asocia a peores resultados e impacto negativo en la sobrevida. ${ }^{29}$

En el presente estudio debemos considerar varias limitaciones. Es un estudio retrospectivo de una única institución, donde la mayoría de los pacientes corresponden a los alrededores de una de las localidades con los índices más altos de violencia en Bogotá. No evaluamos los pacientes que ingresaron muertos al hospital, solamente los que llegaron vivos y fueron intervenidos quirúrgicamente, lo que haría que nuestra mortalidad global ascendiera si se incluyeran datos de estudios postmortem. Sin embargo reconocemos que el poder estadístico de la serie es importante, por el tamaño de la muestra, los resultados del taponamiento cardiaco y la sobrevida hospitalaria.

\section{CONCLUSIONES}

El trauma cardiaco penetrante en nuestro medio es causado principalmente por heridas de arma cortopunzante, lo que explica la sobrevida de un $15 \%$ de nuestra serie. Nuestro estudio reúne 240 heridas cardiacas penetrantes durante un periodo de 10 años, lo que nos coloca entre una de las mas grandes de Colombia y del mundo. Entre los factores de riesgo de mortalidad en heridas cardiacas penetrantes encontramos las heridas por arma de fuego y el compromiso hemodinámico. Las tasas de mortalidad de nuestros pacientes son similares a los descritos en centros de trauma de los Estados Unidos, Europa y Colombia e indican que nuestra institución cumplen con protocolos bien definidos de atención de los pacientes con trauma penetrante de corazón. Se requieren estudios adicionales en donde se relacionen el transporte prehospitalario, la utilidad de la toracotomía de resucitación en nuestro medio, los costos y riesgos de su implementación.

\section{REFERENCIAS}

1. Krug EG, Dahlberg LL, Mercy JA, Zwi AB, Lozano R (Eds). World report on violence and health. Geneva: World Health Organization 2002.

2. Instituto Nacional de Medicina Legal y Ciencias Forenses, Grupo Centro de Referencia Nacional Sobre Violencia. Forensis Datos Para la Vida 2011;12(1):10-16. 
3. Mandal A, Sanusi M. Penetrating chest wounds: 24 years experience. World Journal of Surgery 2001;25(9):1145-49.

4. Rhee PM, Foy H, Kaufmann C, Areola C, Boyle E, et al. Penetrating cardiac injuries: A population-based study. Journal of Trauma 1980;45(2):366-70.

5. Knott-Craig C, Dalton R, Rossouw G, Barnard P. Penetrating cardiac trauma: Management strategy based on 129 surgical emergences over 2 years. Annals of Thoracic Surgery 1992;53(6): 1006-09.

6. Henderson V, Smith R, Fry W, Morabito D, Peskin G, et al. Cardiac injuries: Analysis of an selected series of 251 cases. Journal of Trauma 1994;36(3):341-48.

7. Fallahnejad M, Kutty A, Wallace HW. Secondary lesions of penetrating cardiac injuries. Annals of Surgery 1980;191(2): 228-33.

8. Mattox KL, Limacher MC, Feliciano DV, Colosimo L, O’Meara ME, Bealli AC Jr, DeBakey ME. Cardiac evaluation following heart injury. Journal of Trauma 1985;25(8):758-65.

9. Asensio JA, Murray J, Demetriades D, et al. Penetrating cardiac injuries: A prospective study of variables predicting outcomes. Journal of the American College of Surgeons 1998;186(1):24-34

10. Kang N, Hsee L, Rizoli S, Alison P. Penetrating cardiac injury: Overcoming the limits set by Nature. Injury 2009;40(9):919-27.

11. Cámara de comercio de Bogotá, Observatorio de seguridad en Bogotá 2010;39:3-42.

12. Ivatury R. Injury to the heart. In: Trauma (3rd ed). Appleton \& Lange 1996:409-21.

13. American College of Surgeons, Committee on Trauma. Advanced Trauma Life Support Manual. Chicago, IL 1997.

14. Mitchell ME, Muakkassa FF, Poole GV, et al. Surgical approach of choice for penetrating cardiac wounds. Journal of Trauma 1993;34(1):17-20.

15. Beck C. Two cardiac compression triads. Journal of the American Medical Association 1935;104:714-16.

16. Moore EE, Malangoni MA, Cogbill TH. Organ injury scaling IV: Thoracic, vascular, lung, cardiac and diaphragm. Journal of Trauma 1994;36(3):299-300.

17. Emura AF, Coral O. Injuria cardíaca penetrante: Índices de trauma vs mortalidad. Revista Colombiana de Cirugía 1997;12(1): $4-11$.

18. Villegas MI, Morales CH, Rosero E, Benítez G, Restrepo FC, Fernández IM, et al. Trauma Cardíaco penetrante: Factores pronósticos. Revista Colombiana de Cirugía. 2007;22(3):148-56.

19. Buckman RF Jr, Badellino MM, Mauro LH, Asensio JA, Caputo C, Gass J, Grosh JD. Penetrating cardiac wounds: Prospective study of factors influencing initial resuscitation. Journal of Trauma 1993;34(5):717-25.

20. Campbell NC, Thomson SR, Muckart DJ, Meumann CM, Van Middelkoop I, Botha JB. Review of 1198 cases of penetrating cardiac trauma. British Journal of Surgery 1997;84(12):1737-40.

21. Rashid MA, Wikstrom T, Ortenwall P. Cardiac injuries: A 10-year experience. The European Journal of Surgery 2000;166(1):22-28.
22. Rashid MA, Lund JT. Trauma to the heart and thoracic aorta: The Copenhagen experience. Interactive Cardiovascular and Thoracic Surgery 2003;2(1):53-57.

23. Moreno C, Moore EE, Majure JA, et al. Pericardial tamponade: a critical determinant for survival following penetrating cardiac wounds. Journal of Trauma 1986;26(9):821-25.

24. Mitchell ME, Muakkassa FF, Poole GV, et al. Surgical approach of choice for penetrating cardiac wounds. Journal of Trauma 1993;34(1):17-20.

25. Degiannis E, Loogna P, Doll D, Bonanno F, Bowley DM, Smith MD. Penetrating Cardiac Injuries: Recent experience in South Africa. World Journal of Surgery. 2006;30(7):1258-64.

26. Gao J, Gao YH, Wei GB. Penetrating cardiac wounds: Principles for surgical management. World Journal of Surgery 2004;28(10): 1025-29.

27. Demetriades D, Charalambides D, Sareli P, et al. Late sequelae of penetrating cardiac injuries. British Journal of Surgery 1990; 77(7):813-14

28. Molina EJ, Gaughan JP, Kulp H, McClurken JB, Goldberg AJ, Seamon MJ. Outcomes after emergency department thoracotomy for penetrating cardiac injuries: A new perspective. Interactive Cardio Vascular and Thoracic Surgery 2008;7(5):845-49.

29. Seamon MJ, Fisher CA, Gaughan J, Lloyd M, Bradley KM, Santora TA. Prehospital procedures before emergency department thoracotomy: 'Scoop and run' saves lives. Journal of Trauma 2007;63(1):113-20.

\section{ACERCA DE LOS AUTORES}

\section{Andrés Isaza-Restrepo (Correspondencia)}

Profesor Titular Cirugía General Universidad del Rosario Hospital Occidente de Kennedy, Bogota, Colombia, e-mail: mafejimenez2000@yahoo.es

\section{Dínimo José Bolívar-Sáenz}

Residente de Cirugía General 4to año. Universidad del Rosario Hospital Occidente de Kennedy, Bogota, Colombia

\section{Marcos Tarazona}

Instructor Asistente Cirugía General Universidad del Rosario, Hospital Occidente de Kennedy, Bogota, Colombia

\section{María Fernanda Jiménez}

Profesor Titular Cirugía General Universidad del Rosario, Hospital Occidente de Kennedy, Bogota, Colombia

\section{Fabián Armando Gil Laverde}

Bioestadístico, Profesor Asistente Departamento de Epidemiología Clínica y Bioestadística, Pontificia Universidad Javeriana, Hospital Occidente de Kennedy, Bogota, Colombia 